\title{
Continuous Non-singular Fast Terminal Sliding Mode Control for an Active Gravity Field Simulator
}

\author{
Jiao Jia, Yingmin Jia*, Shihao Sun \\ The Seventh Research Division and the Center for Information and Control \\ School of Automation Science and Electrical Engineering \\ Beihang University (BUAA), 37 Xueyuan Road, Haidian District \\ Beijing, 100191, China \\ E-mail:annebuaa@hotmail.com;ymjia@buaa.edu.cn; jxcrssh@126.com \\ www.buaa.edu.cn
}

\begin{abstract}
In this paper, a global non-singular fast terminal sliding mode controller (FTSMC) is designed for an active gravity field simulator (AGFS). The stability of the control algorithm can be easily verified by using Lyapunov theory. It is shown that the proposed controller can eliminate the chattering effect without losing the robustness property. Simulation results show that faster and high-precision tracking performance can be obtained compared with the conventional continuous sliding mode control method.
\end{abstract}

Keywords: gravity field simulator, fast terminal sliding mode, robustness, finite-time stability

\section{Introduction}

Gravity field simulators are necessary equipment for spacecraft ground verification such as space manipulators, solar array deployments, lunar rovers and so on. The control objectives are to keep these spacecraft vertically and the gravity of the objects are partly or completely compensated as demanded. And the dynamics of the simulators are nonlinear coupling system with interferences and uncertainties. It is now known that terminal sliding mode (TSM) control $^{1-4}$ as an effective robust control method has been effectively applied to the control of nonlinear systems such as robotic manipulators, electrical motors and power systems. TSM is a finite time stability control strategy, which can stabilize system states to their equilibrium points or track the desired trajectory in finite time. Due to some superior properties, such as fast convergence, strong robustness, and high precision, TSM has attracted extensive attention in control theory studies and in practical applications. In this paper, a non- singular fast terminal sliding mode controller is applied to an AGFS.

The rest of this paper is organized as follows. The simulation mechanism of the platform is introduced in section 2. In section 3, a NFTSMC is developed and the stability is proved. Simulation examples are given to demonstrate the effectiveness of the proposed control algorithms in section 4. Finally, we end this paper with some conclusions.

\section{Simulation Mechanism}

The simulation platform ${ }^{5}$ is a servo system consisting of an $\mathrm{x}$-axis direction mechanism driven by the $\mathrm{x}$-axis direction motor, a $y$-axis direction mechanism driven by the $y$-axis direction motor, a z-axis direction mechanism driven by the $\mathrm{z}$-axis direction motor, a universal joint, a spring buffer, a dual-axis tilt sensor, a tension sensor and an unconstrained suspension section (USS). As described in Fig. 1 the object is connected to the platform through the USS. The USS is linked to the spring buffer. The spring buffer is attached to the universal joint. The universal joint is connected to the $\mathrm{z}-$ axis direction mechanism through a tension sensor to

* Corresponding author. 
detect the force between them. A dual-axis tilt sensor is mounted on the spring buffer to determine the angle of the spring buffer with respect to the vertical axis or $\mathrm{z}$ axis direction.
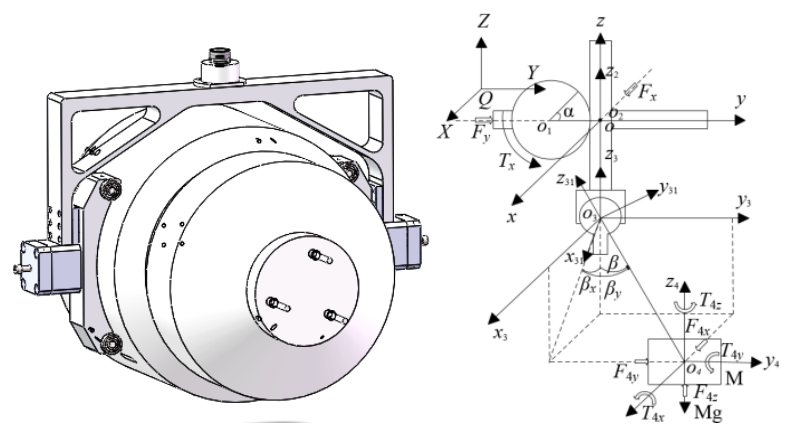

Fig. 1. The USS and coordinate system of the AGFS

\section{Continuous FTSMC}

The dynamics of the $\mathrm{AGFS}^{5}$ can be written as

$$
\boldsymbol{M}_{m} \ddot{\boldsymbol{q}}_{2}+\boldsymbol{C} \dot{\boldsymbol{q}}_{2}+\boldsymbol{G}=\boldsymbol{F}_{1}-\boldsymbol{M}_{1} \boldsymbol{M}_{2}^{-T} \boldsymbol{F}_{2}
$$

Define $\boldsymbol{M}(\boldsymbol{q})=\boldsymbol{M}_{m}, \boldsymbol{F}=\boldsymbol{F}_{1}, \boldsymbol{F}_{d}=-\boldsymbol{M}_{1} \boldsymbol{M}_{2}^{-T} \boldsymbol{F}_{2}$, and we obtain

$$
\boldsymbol{M}(\boldsymbol{q}) \ddot{\boldsymbol{q}}+\boldsymbol{C}(\boldsymbol{q}, \dot{\boldsymbol{q}}) \dot{\boldsymbol{q}}+\boldsymbol{G}(\boldsymbol{q})=\boldsymbol{F}+\boldsymbol{F}_{d}
$$

where $\boldsymbol{q}, \dot{\boldsymbol{q}}, \ddot{\boldsymbol{q}} \in \boldsymbol{R}^{3}$ are the vectors of position, velocity and acceleration, respectively, $\boldsymbol{M}(\boldsymbol{q})=\boldsymbol{M}_{0}(\boldsymbol{q})+$ $\delta M(q) \in R^{3 \times 3}, \boldsymbol{C}(q, \dot{q})=C_{0}(q, \dot{q})+\delta C(q, \dot{q}), \quad \boldsymbol{G}(\boldsymbol{q})=$ $\boldsymbol{G}_{0}(\boldsymbol{q})+\boldsymbol{\delta} \boldsymbol{G}(\boldsymbol{q}) \in \boldsymbol{R}^{3}, \quad \boldsymbol{F} \in \boldsymbol{R}^{3}$ is the vector of applied torque or equivalent force, $\boldsymbol{F}_{d} \in \boldsymbol{R}^{3}$ is the vector of driving force operating on the object, $\left\|\boldsymbol{F}_{d}\right\| \leq f_{d}$. Here $\boldsymbol{M}_{0}(\boldsymbol{q}), \boldsymbol{C}_{0}(\boldsymbol{q}, \dot{\boldsymbol{q}})$ and $\boldsymbol{G}_{0}(\boldsymbol{q})$ are the nominal parts, whereas $\boldsymbol{\delta} \boldsymbol{M}(\boldsymbol{q}), \boldsymbol{\delta} \boldsymbol{C}(\boldsymbol{q}, \dot{\boldsymbol{q}})$ and $\boldsymbol{\delta} \boldsymbol{G}(\boldsymbol{q})$ represent the perturbations in the system matrices. Then, the dynamical model of the AGFS can be rewritten as

$$
\boldsymbol{M}_{0}(\boldsymbol{q}) \ddot{\boldsymbol{q}}+\boldsymbol{C}_{0}(\boldsymbol{q}, \dot{\boldsymbol{q}}) \dot{\boldsymbol{q}}+\boldsymbol{G}_{0}(\boldsymbol{q})=\boldsymbol{F}+\boldsymbol{F}_{d}+\boldsymbol{T}(\boldsymbol{q}, \dot{\boldsymbol{q}}, \ddot{\boldsymbol{q}})
$$

where $\boldsymbol{T}(\boldsymbol{q}, \dot{\boldsymbol{q}}, \ddot{\boldsymbol{q}})=-\boldsymbol{\delta} \boldsymbol{M}(\boldsymbol{q}) \ddot{\boldsymbol{q}}-\boldsymbol{\delta} \boldsymbol{C}(\boldsymbol{q}, \dot{\boldsymbol{q}}) \dot{\boldsymbol{q}}-\boldsymbol{\delta} \boldsymbol{G}(\boldsymbol{q}) \in \boldsymbol{R}^{3}$ is the lumped system uncertainty, which is bounded by the following function

$$
\|\boldsymbol{T}(\boldsymbol{q}, \dot{\boldsymbol{q}}, \ddot{\boldsymbol{q}})\| \leq b_{0}+b_{1}\|\boldsymbol{q}\|+b_{2}\|\dot{\boldsymbol{q}}\|^{2}
$$

Let $\boldsymbol{q}_{d} \in \boldsymbol{R}^{3}$ be a twice differentiable desired trajectory, and define the tracking error as $\boldsymbol{e}=\boldsymbol{q}-\boldsymbol{q}_{d}$. The control objective is to design a feedback control $\boldsymbol{F}$ such that the AGFS output $\boldsymbol{q}$ tracks the desired trajectory $\boldsymbol{q}_{d}$ in finite time.
Before giving the main results, the following vectors are defined as

$$
\begin{aligned}
& \boldsymbol{x}^{\gamma}=\left[x_{1}^{\gamma_{1}}, \ldots, x_{n}^{\gamma_{n}}\right]^{T},|\boldsymbol{x}|^{\gamma}=\left[\left|x_{1}\right|^{\gamma_{1}}, \ldots,\left|x_{n}\right|^{\gamma_{n}}\right]^{T} \\
& \operatorname{sgn}(\boldsymbol{x})^{\gamma}=\left[\left|x_{1}\right|^{\gamma_{1}} \operatorname{sgn}\left(x_{1}\right), \ldots,\left|x_{n}\right|^{\gamma_{n}} \operatorname{sgn}\left(x_{n}\right)\right]^{T}
\end{aligned}
$$

where $\boldsymbol{x}=\left[x_{1}, \ldots, x_{n}\right]^{T} \in \boldsymbol{R}^{n}$, and $\operatorname{sgn}(\cdot)$ is the sign function.

Hence, the TSM can be defined as

$$
\boldsymbol{s}=\boldsymbol{\alpha} \boldsymbol{e}+\boldsymbol{\beta} \operatorname{sgn}(\dot{\boldsymbol{e}})^{\gamma}=0
$$

where $\boldsymbol{s}=\left[s_{1}, s_{2}, s_{3}\right]^{T} \in \boldsymbol{R}^{3}, \boldsymbol{\alpha}=\operatorname{diag}\left(\alpha_{1}, \alpha_{2}, \alpha_{3}\right), \boldsymbol{\beta}=$ $\operatorname{diag}\left(\beta_{1}, \beta_{2}, \beta_{3}\right)$ and $1<\gamma_{1}, \gamma_{2}, \gamma_{3}<2$. For simplicity, we choose $\gamma=\gamma_{1}=\gamma_{2}=\gamma_{3}$. The reaching law is defined as

$$
\dot{\boldsymbol{s}}=-\boldsymbol{k}_{1} \boldsymbol{s}-\boldsymbol{k}_{2} \operatorname{sgn}(\boldsymbol{s})^{\boldsymbol{\rho}}
$$

where $\boldsymbol{k}_{1}=\operatorname{diag}\left(k_{11}, k_{12}, k_{13}\right), \boldsymbol{k}_{2}=\operatorname{diag}\left(k_{21}, k_{22}, k_{23}\right)$, $0<\rho=\rho_{1}=\rho_{2}=\rho_{3}<1$.

Theorem 1. For the AGFS (3), if the TSM manifold is defined as (5), the reaching law is chosen as (6) and the continuous FTSM control is designed as

$$
\begin{aligned}
\boldsymbol{F}= & \boldsymbol{F}_{0}+\boldsymbol{F}_{1}, \\
\boldsymbol{F}_{0}= & \boldsymbol{C}_{0}(\boldsymbol{q}, \dot{\boldsymbol{q}}) \dot{\boldsymbol{q}}+\boldsymbol{G}_{0}(\boldsymbol{q})+\boldsymbol{M}_{0}(\boldsymbol{q}) \ddot{\boldsymbol{q}}_{d} \\
& -\boldsymbol{\alpha} \boldsymbol{M}_{0}(\boldsymbol{q}) \boldsymbol{\beta}^{-1} \boldsymbol{\gamma}^{-1} \operatorname{sgn}(\dot{\boldsymbol{e}})^{2-\gamma} \\
\boldsymbol{F}_{1}= & -\boldsymbol{M}_{0}(\boldsymbol{q})\left(\boldsymbol{k}_{1} \boldsymbol{s}+\boldsymbol{k}_{2} \operatorname{sgn}(\boldsymbol{s})^{\boldsymbol{\rho}}\right)
\end{aligned}
$$

where $\gamma=\operatorname{diag}(\gamma, \gamma, \gamma)$. Then the system trajectory will converge to the neighborhood of $\mathbf{s}=0$ as

$$
\begin{gathered}
\|\boldsymbol{s}\| \leq \Delta=\min \left\{\Delta_{1}, \Delta_{2}\right\}, \\
\Delta_{1}=\left\|\boldsymbol{M}_{0}^{-1}(\boldsymbol{q})\right\|\left(b_{0}+b_{1}\|\boldsymbol{q}\|+b_{2}\|\dot{\boldsymbol{q}}\|^{2}+f_{d}\right) / \underline{k}_{1} \\
\Delta_{2}=\left(\left\|\boldsymbol{M}_{0}^{-1}(\boldsymbol{q})\right\|\left(b_{0}+b_{1}\|\boldsymbol{q}\|+b_{2}\|\dot{\boldsymbol{q}}\|^{2}+f_{d}\right) / \underline{k}_{2}\right)^{1 / \rho}
\end{gathered}
$$

in finite time, where $\underline{k}_{1}=\min _{i}\left\{k_{1 i}\right\}>0$, and $\underline{k}_{2}=$ $\min _{i}\left\{k_{2 i}\right\}>0$. Furthermore, the tracking errors $\boldsymbol{e}$ and $\dot{\boldsymbol{e}}$ will converge to the regions

in finite time.

$$
\begin{aligned}
& \left|e_{i}\right| \leq \Delta_{e}=2 \Delta / \alpha_{i} \\
& \left|\dot{e}_{i}\right| \leq \Delta_{\dot{e}}=\left(\Delta / \beta_{i}\right)^{1 / \gamma_{i}}
\end{aligned}
$$

Proof. Consider a Lyapunov candidate function as:

$$
V=\frac{1}{2} \boldsymbol{s}^{T} \boldsymbol{s}
$$

By differentiating $V$ with respect to time, substituting (6) and (7) into it, we have 
$\dot{V}=-\boldsymbol{s}^{T} \boldsymbol{\beta} \gamma \operatorname{diag}\left(|\dot{\boldsymbol{e}}|^{\gamma-1}\right)\left(\boldsymbol{k}_{1} \boldsymbol{s}+\boldsymbol{k}_{2} \operatorname{sgn}(\boldsymbol{s})^{\boldsymbol{\rho}}-\boldsymbol{M}_{0}^{-1}(\boldsymbol{q})\left(\boldsymbol{T}+\boldsymbol{F}_{d}\right)(11)\right.$

which can be further changed into the following two forms:

$$
\begin{aligned}
\dot{V}= & -\boldsymbol{s}^{T} \boldsymbol{\beta} \gamma \operatorname{diag}\left(\mid \dot{\boldsymbol{e}}^{\gamma-1}\right)\left(\left(\boldsymbol{k}_{1}-\operatorname{diag}\left(\boldsymbol{M}_{0}^{-1}(\boldsymbol{q})\right.\right.\right. \\
& \left.\left.\left.\times\left(\boldsymbol{T}+\boldsymbol{F}_{d}\right)\right) \operatorname{diag}^{-1}(\boldsymbol{s})\right) \boldsymbol{s}+\boldsymbol{k}_{2} \operatorname{sgn}(\boldsymbol{s})^{\boldsymbol{\rho}}\right) \\
\dot{V}=- & \boldsymbol{s}^{T} \boldsymbol{\beta} \gamma \operatorname{diag}\left(|\dot{\boldsymbol{e}}|^{\gamma-1}\right)\left(\boldsymbol{k}_{1} \boldsymbol{s}+\left(\boldsymbol{k}_{2}-\operatorname{diag}\left(\boldsymbol{M}_{0}^{-1}(\boldsymbol{q})\right.\right.\right. \\
& \left.\left.\left.\times\left(\boldsymbol{T}+\boldsymbol{F}_{d}\right)\right) \operatorname{diag}^{-1}\left(\operatorname{sgn}(\boldsymbol{s})^{\boldsymbol{\rho}}\right)\right) \operatorname{sgn}(\boldsymbol{s})^{\boldsymbol{\rho}}\right)
\end{aligned}
$$

For Eq. (11), if we can keep the matrix $\boldsymbol{k}_{1}-\operatorname{diag}\left(\boldsymbol{M}_{0}^{-1}(\boldsymbol{q})\left(\boldsymbol{T}+\boldsymbol{F}_{d}\right)\right) \operatorname{diag}^{-1}(\boldsymbol{s})$ positive definite, the structure

$$
\dot{V}=-\boldsymbol{s}^{T} \underline{\boldsymbol{k}}_{1} \boldsymbol{s}-\boldsymbol{s}^{T} \underline{\boldsymbol{k}}_{2} \operatorname{sig}(\boldsymbol{s})^{\rho}
$$

is kept and $\underline{\boldsymbol{k}}_{1}=\boldsymbol{\beta} \boldsymbol{\gamma} \operatorname{diag}\left(|\dot{\boldsymbol{e}}|^{\gamma-1}\right)\left(\boldsymbol{k}_{1}-\operatorname{diag}\left(\boldsymbol{M}_{0}^{-1}(\boldsymbol{q})(\boldsymbol{T}+\right.\right.$ $\left.\left.\left.\boldsymbol{F}_{d}\right)\right) \operatorname{diag}^{-1}(\boldsymbol{s})\right), \underline{\boldsymbol{k}}_{2}=\boldsymbol{\beta} \boldsymbol{\gamma} \operatorname{diag}\left(\mid \dot{\boldsymbol{e}}^{\gamma-1}\right) \boldsymbol{k}_{2}$. It can be proved that

$$
\dot{V} \leq-2 \underline{k_{1}} V-2^{(\rho+1) / 2} \underline{k}_{2} V^{(\rho+1) / 2}
$$

and the settling time is

$$
T \leq \frac{\ln \left(\left(\underline{k}_{1} V^{(1-\rho) / 2}+2^{(\rho-1) / 2} \underline{k}_{2}\right) / 2^{(\rho-1) / 2} \underline{k}_{2}\right.}{(1-\rho) \underline{k}_{2}}
$$

therefore finite-time stability is guaranteed. Assume $\boldsymbol{M}_{0}^{-1}(\boldsymbol{q})\left(\boldsymbol{T}+\boldsymbol{F}_{d}\right)=\boldsymbol{Q}=\left[Q_{1}, Q_{2}, Q_{3}\right]^{T} \in \boldsymbol{R}^{3}$; then if $\underline{k}_{1}-$ $\left|Q_{i}\right| /\left|s_{i}\right|>0$, the region

$$
\begin{gathered}
\left|s_{i}\right| \leq\left|Q_{i}\right| / \underline{k}_{1} \\
\|\boldsymbol{s}\| \leq\left\|\boldsymbol{M}_{0}^{-1}(\boldsymbol{q})\right\|\left(b_{0}+b_{1}\|\boldsymbol{q}\|+b_{2}\|\dot{\boldsymbol{q}}\|^{2}+f_{d}\right) / \underline{k}_{1}
\end{gathered}
$$

can be reached infinite time. For Eq. (13), by similar analysis for (12), we can have that the region

$$
\begin{gathered}
\left|s_{i}\right|^{\rho} \leq\left|Q_{i}\right| / \underline{k}_{2} \\
\|\boldsymbol{s}\| \leq\left(\left\|\boldsymbol{M}_{0}^{-1}(\boldsymbol{q})\right\|\left(b_{0}+b_{1}\|\boldsymbol{q}\|+b_{2}\|\dot{\boldsymbol{q}}\|^{2}+f_{d}\right) / \underline{k}_{2}\right)^{1 / \rho}
\end{gathered}
$$

can be reached infinite time.

Synthesizing inequalities (17) and (18), we have that the region (8) can be reached infinite time. We can explain $\dot{\boldsymbol{e}}_{i}=0$ does not hinder the finite-time reachability of the region (17) by substituting the control (7) into (3).

\section{Simulation}

The nominal values are $\hat{m}_{1}=0.5 \mathrm{~kg}, \hat{m}_{2}=4 \mathrm{~kg}, \hat{m}_{0 x}$ $=16 \mathrm{~kg}, \quad \hat{m}_{0 y}=40 \mathrm{~kg}, \hat{M}=10 \mathrm{~kg}, \hat{k}=500 \mathrm{~N} / \mathrm{m}, \quad \hat{l}_{0}=1 \mathrm{~m}$, $\hat{g}=10 \mathrm{~m} / \mathrm{s}^{2}, \hat{R}=0.02 \mathrm{~m}$. The parameter values are $m_{1}=0.55 \mathrm{~kg}, m_{2}=4.4 \mathrm{~kg}, m_{0 x}=17.6 \mathrm{~kg}, m_{0 y}=44 \mathrm{~kg}, M=$ $11 \mathrm{~kg}, k=550 \mathrm{~N} / \mathrm{m}, l_{0}=1.1 \mathrm{~m}, g=9.8 \mathrm{~m} / \mathrm{s}^{2}, R=0.022 \mathrm{~m}$.
The bound parameters of system uncertainties in (4) are assumed to be $b_{0}=0.8, b_{1}=1, b_{2}=8$.

The reference signals are given by $\boldsymbol{q}_{d}=[u(t), 0,0]^{T}$ according to the simulation mechanism of AGFS and $u(\mathrm{t})$ represent offload quantity of the object gravity. When $u(\mathrm{t})=0$ the object's gravity is compensated completely and when $u(t)=0.2$ the object's gravity is at normal situation. When the value of $u(\mathrm{t})$ is between $(0,0.2)$, the object's gravity is partly compensated and when $u(\mathrm{t})$ is negative, the object's gravity is greater than earth's gravity.

Firstly, define $\boldsymbol{\alpha}=\mathbf{I}$ corresponding to the controller designed in reference [2].

The object driving input is shown in Fig.2 which is regarded as the system disturbance.

Fig. 3 shows the system output, system output error, the sliding surface function and system control input when $u(t)=\varepsilon(t)$ with TSM controller.

$$
\varepsilon(t)=\left\{\begin{array}{lr}
0.2 \sin (20 t) & t \leq \pi / 40 \\
0.2 & t>\pi / 40
\end{array}\right.
$$

Fig. 4 shows the system output, system output error, the sliding surface function and system control input when $u(t)=\varepsilon(t)$ with controller (7).

Fig. 5 shows the system output with FTSMC when the object gravity varies according to the law role of the gravity effect.

For the uncertain system with bounded disturbances and noises, the control parameters of the TSM controller are chosen according to (8) as

$$
\begin{gathered}
\boldsymbol{k}_{1}=\operatorname{diag}(2500,8000,8000), \boldsymbol{k}_{2}=\operatorname{diag}(2000,2000,2000), \\
\gamma=1.6, \rho=1 / 3, \boldsymbol{\beta}=\operatorname{diag}(0.4,5,5)
\end{gathered}
$$

for $u(t)=\varepsilon(t)$.

The boundary layer $\|s\| \leq 2.2 \times 10^{-3}$ is firstly reached in the finite time $t=0.2 \mathrm{~s}$ and $\|\boldsymbol{e}\| \leq 2.3 \times 10^{-3}$ is reached in the finite time $t=0.5 \mathrm{~s}$ as shown in Fig. 3 .

The control parameters of the FTSMC are chosen according to (8) as

$$
\begin{gathered}
\boldsymbol{k}_{1}=\operatorname{diag}(2000,2000,2000), \boldsymbol{k}_{2}=\operatorname{diag}(2000,2000,2000), \\
\gamma=1.6, \rho=1 / 3, \boldsymbol{\alpha}=\operatorname{diag}(12,10,10), \boldsymbol{\beta}=\operatorname{diag}(0.4,5,5)
\end{gathered}
$$

for $u(t)=\varepsilon(t)$.

The simulation results shown in Fig. 4 verify the robustness of the FSTM approach. The boundary layer $\|\boldsymbol{s}\| \leq 1.5 \times 10^{-3}$ is firstly reached in the finite time $t=0.17 \mathrm{~s}$ and $\|\boldsymbol{e}\| \leq 1.5 \times 10^{-4}$ is reached in the finite time $t=0.18 s$. 


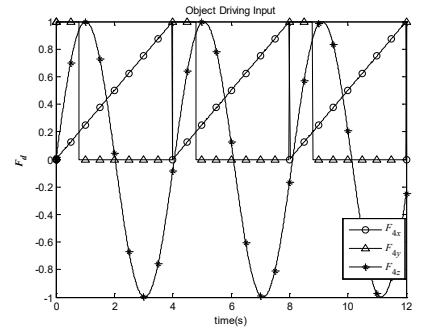

Fig. 2. The object driving input
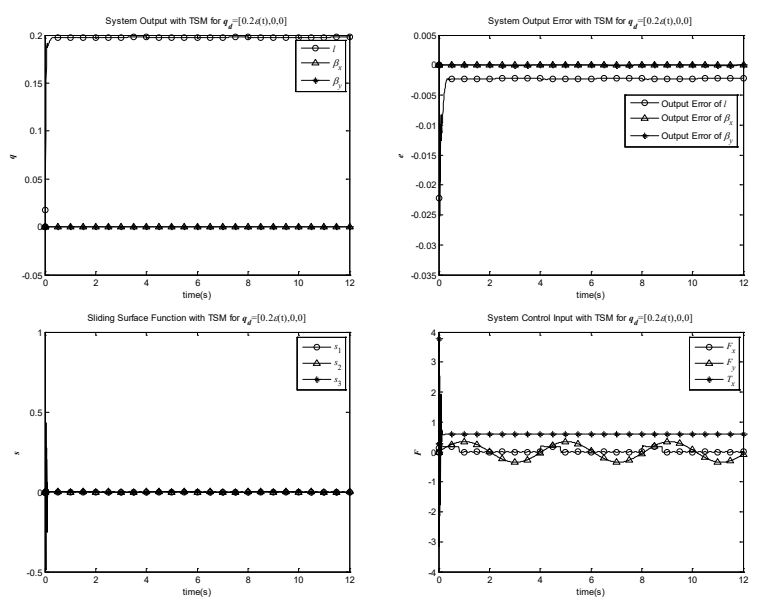

Fig. 3. TSM control of the AGFS when $u(t)=\varepsilon(t)$
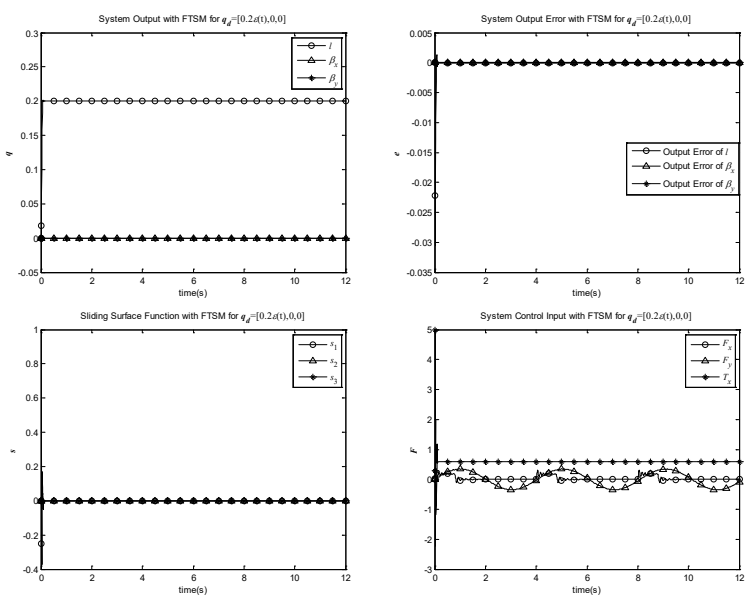

Fig. 4. FTSMC control of the AGFS when $u(t)=\varepsilon(t)$

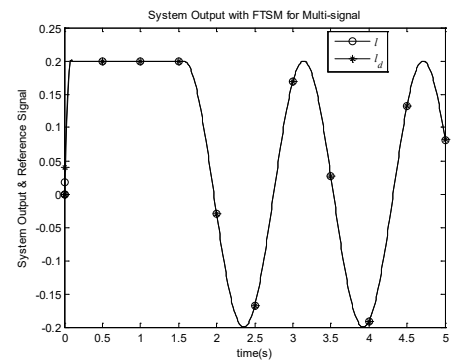

Fig. 5. FTSMC control of the AGFS
With the same control parameters of the FTSMC, Fig. 5 shows the system can track time-varying trajectory with $\|\boldsymbol{e}\| \leq 2 \times 10^{-4}$ when $t \geq 0.13 \mathrm{~s}$.

Therefore much smaller output error and faster convergence speed are obtained with the FTSMC method.

\section{Conclusions}

In this paper, a new fast terminal sliding mode controller is developed for the AGFS with the finitetime convergent property. The proposed control algorithms can guarantee the tracking errors converge to arbitrarily small value. And the developed control approach can be used to a more general Lagrange-like second-order nonlinear system.

\section{Acknowledgements}

This work was supported by the NSFC (61327807, 61521091, 61520106010, 61134005) and the National Basic Research Program of China (973 Program: 2012CB821200, 2012CB821201)

\section{References}

1. Y. Feng, et al., Non-singular terminal sliding mode control of rigid manipulators, J. Automatica. 38(12) (2002) 2159-2167.

2. S. Yu, et al., Continuous finite-time control for robotic manipulators with terminal sliding mode, J. Automatica. 41(11) (2005)1957-1964.

3. M. B. R. Neila and D. Tarak, Adaptive terminal sliding mode control for rigid robotic manipulators, $J$. International Journal of Automation and Computing. 8(2) (2011) 215-220.

4. D. Zhao, et al., A new terminal sliding mode control for robotic manipulators, J. International Journal of Control. 82(10) (2009) 1804-1813.

5. J. Jia, et al., Modeling and Control of a Suspended Gravity Compensation System with Rigid-Elastic Coupling, J. Journal of Robotics, Networking and Artificial Life2016, 3(2) (2016)79-83. 